BIOFILMS

\title{
Building up the matrix
}

\section{cef \\ novel insights \\ into the assembly and \\ functionality of the biofilm matrix}

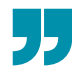

Biofilms are formed by bacterial communities embedded in a self-produced extracellular matrix that comprises a complex mixture of polysaccharides, lipids, extracellular DNA (eDNA) and proteins, and protects bacteria from environmental stresses, exposure to antibiotics and immune attack.

Two independent recent studies have revealed how distinct components of the biofilm matrix contribute to its architectural stability and functionality.

Arnaouteli, Ferreira, Schor et al. showed two distinct functional roles for the Bacillus subtilis BslA biofilm matrix protein. BslA is an amphipathic protein that was previously shown to contribute to the high hydrophobicity of $B$. subtilis biofilms. BslA can have two structural forms, as it includes a hydrophobic cap that can be either hidden in the interior of the protein in aqueous environments or can be exposed in hydrophobic environments, such as the air-water interface present at the surface of biofilms. This study revealed that BslA can also assume two functional forms, a monomer or a dimer, and that the form that is adopted

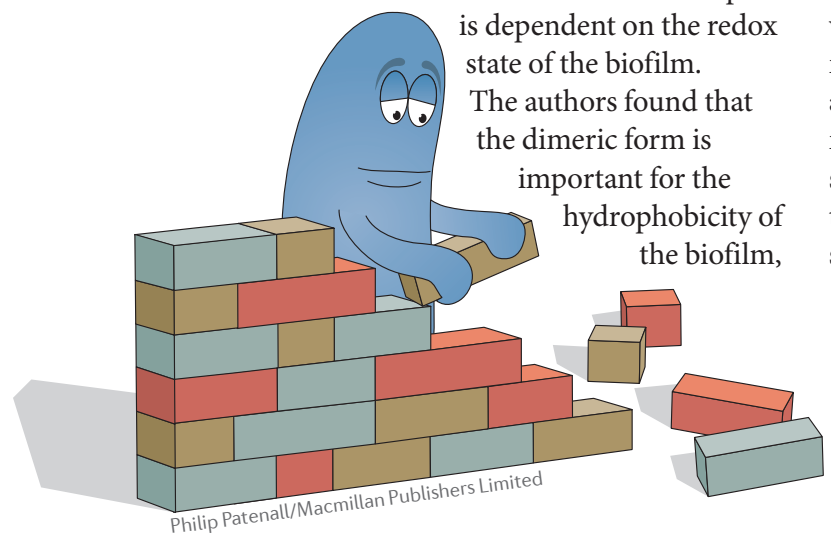

whereas the monomer is important for biofilm architecture. Two key cysteine residues that are present in the carboxy-terminal domain were shown to be involved in the formation of disulfide bonds and thus dimer formation. The authors propose that disulfide bond formation is driven by a combination of spontaneous and catalytic activity regulated by the thiol-disulfide oxidoreductases BdbA and BdbD. Moreover, they showed that the upper region of the biofilm was more oxidizing than the base owing to the oxygen-rich conditions; these conditions could promote disulfide bond formation and the dimerization of BslA. By contrast, the anoxic conditions at the base of the biofilm might promote the monomeric form of BslA, which was shown to be important for the maintenance of the architectural complexity of biofilm.

In a second study, Jurcisek et al. identified a novel active mechanism in Haemophilus influenzae that is involved in the release of eDNA and associated DNABII proteins into the extracellular space for incorporation into the biofilm matrix. The authors showed that this active mechanism worked independently from other mechanisms such as cell lysis. In addition, fluorescence time-lapse microscopy and scanning transmission microscopy analyses showed that release occurred at a discrete subpolar location in the bacterial outer membrane. This extracellular release required a two-step transport mechanism that involves two distinct bacterial protein systems. Indeed, the first stage of the transport from the cytoplasm across the inner membrane and into the periplasmic space was shown to be mediated by an inner membrane pore complex composed of TraC and TraG, which has homology to type IV secretion-like systems. Moreover, the trafficking of eDNA and DNABII proteins from the periplasm across the outer membrane and into the extracellular space was mediated by the outer membrane pore ComE, through which the type-IV pilus is typically threaded. Finally, the authors reported that conditions that induce competence increased the frequency of the extracellular release of DNA.

Taken together, these studies provide novel insights into the assembly and functionality of the biofilm matrix. These findings will provide the basis for developing novel therapeutics that can effectively target components of the biofilm matrix and modulate biofilm stability.

Irene Vacca

ORIGINAL ARTICLES Arnaouteli, S., Ferreira, A. S Schor, M. et al. Bifunctionality of a biofilm matrix protein controlled by redox state. Proc. Natl Acad. Sci. USA http://dx.doi.org/10.1073/pnas.

1707687114 (2017) | Jurcisek, J. A. et al. Nontypeable Haemophilus influenzae releases DNA and DNABII proteins via a T4SS-like complex and ComE of the type IV pilus machinery. Proc. Natl Acad. Sci. USA http://dx.doi.org/10.1073/pnas.1705508114 (2017) 